\title{
A COMPARISON OF THE DELAY SPREAD OBTAINED WITH DIFFERENT POWER DELAY PROFILES DE-NOISING TECHNIQUES
}

\author{
Leni J. de Matos $^{l}$ \\ Bianca S. Marinho
}

\begin{abstract}
Power delay profiles are determined by the processing of data from measurements carried out in a wideband channel. They are contaminated by the channel and the receiver noise and then they need to be de-noised in order to determine the dispersion parameters of the channel. In this paper, three different de-noising techniques are applied to choose the valid multipath in the noisy power delay profiles. The delay spread parameter is determined and the results are evaluated by comparing them with those obtained with the dirty profiles.
\end{abstract}

Keywords: delay spread; indoor propagation; de-noising techniques; CLEAN technique

\section{INTRODUCTION}

Wideband is an important characteristic in the projects of systems since they work with high rates of information. In order to guarantee such rates it is necessary to know time dispersion characteristics of the wideband channel such as delay spread and coherence bandwidth [1]. Herein, the delay spread is calculated from the Power Delay Profiles (PDP) obtained from the processing of acquired data in a channel where the frequency sweep technique was adopted for sounding it. These profiles correspond to multipaths that arrive at a receiver with different delays, amplitudes and phases, but contaminated with an undesirable channel noise and also a receiver noise as well. Consequently, all profiles must be de-noised in order to get more reliable dispersion parameters. Then, a de-noising technique must be applied so that only valid multipaths are considered for evaluating parameters.

Several papers [2]-[5] deal with different de-noising techniques in order to improve PDPs estimates. The purpose of this paper is to apply three of those techniques in PDPs of sounded wideband channels in order to compare the delay spread obtained with each one.

This paper is organized as follows. In Section II, the de-noising techniques are briefly described. In Section III, the measurement setup and its specifications are presented. In Section IV, the results are presented and discussed, including graphs and tables. Finally, in Section V, conclusions are outlined and some suggestions for future research are presented.

\section{DE-NOISING TECHNIQUES}

One of the de-noising techniques deals with CFAR (Constant False Alarm Rate) as presented by Elvino [2]; another one is called CLEAN technique [3] and the wavelet-based de-noising [4] is the third technique.

On the first technique [2], the level of the thermal noise in each profile is estimated using the median of the samples from the dirty PDP, since the average can be distorted significantly due to strong amplitudes of impulsive noise. The CFAR technique provides as a result a value for the noise threshold for each profile which is equal to the

\footnotetext{
1 Universidade Federal Fluminense, Programa de Mestrado em Engenharia de Telecomunicações, e-mail: lenijm@ig.com.br

2 Universidade Federal Fluminense, Programa de Iniciação Científica em Engenharia de Telecomunicações, e-mail: bianca.s.marinho@gmail.com
} 
median of their samples subtracted from the standard deviation of the thermal noise, assumed to be Gaussian, and increased by a guard margin in such a way that separates the impulsive noise of the valid multipath. So, in a PDP, any peak, in which the previous and subsequent samples are below that noise threshold, is considered as impulsive noise and it is discarded. Otherwise, the peak is a valid multipath if the previous and/or subsequent sample is above the noise threshold, but below that peak level. The guard margin corresponds to a false alarm probability fixed value, i.e., the probability of the noise to overcome the threshold of any sample is constant. Herein, this value was set at $5 \times 10^{-6}$ and it means that for each 200.000 measured samples one is disregarded, providing a guard margin of $13.876 \mathrm{~dB}$.

On the CLEAN technique [3], a reference PDP was measured in an environment without scattereres, on Line-of-Sight (LOS) condition, where only the effects of the transceiver setup were considered. The so-called strongest signal is obtained by correlating the PDP received with the reference PDP (without multipath) in order to identify the highest correlation peak. The sample which has generated the highest peak is subtracted from the received PDP resulting in a clean signal which will be correlated again to the reference signal. This procedure is repeated until the level of the "clean" signal reaches the noise threshold. It is considered valid any multipath that overcomes $80 \%$ of correlation.

The wavelet-based de-noising technique [4] is based on wavelets decomposition and provides a softened PDP. The cleaned PDPs showed very low power levels in the tail, then a noise threshold was also regarded in this case and it was valid any peak (multipath) above this threshold.

This technique was independently applied to real and imaginary parts of the complex signal. Dias [4] showed better results in this situation and applying the symlet 8 wavelets, available in the MATLAB software tools.

\section{MEASUREMENT SETUP}

The frequency channel sounding used a vectorial network analyzer (VNA) that swept the 910-1760 MHz band in 1601 frequency samples. Fig. 1 shows the sounding setup [6] used and Table I shows the main characteristics of it.

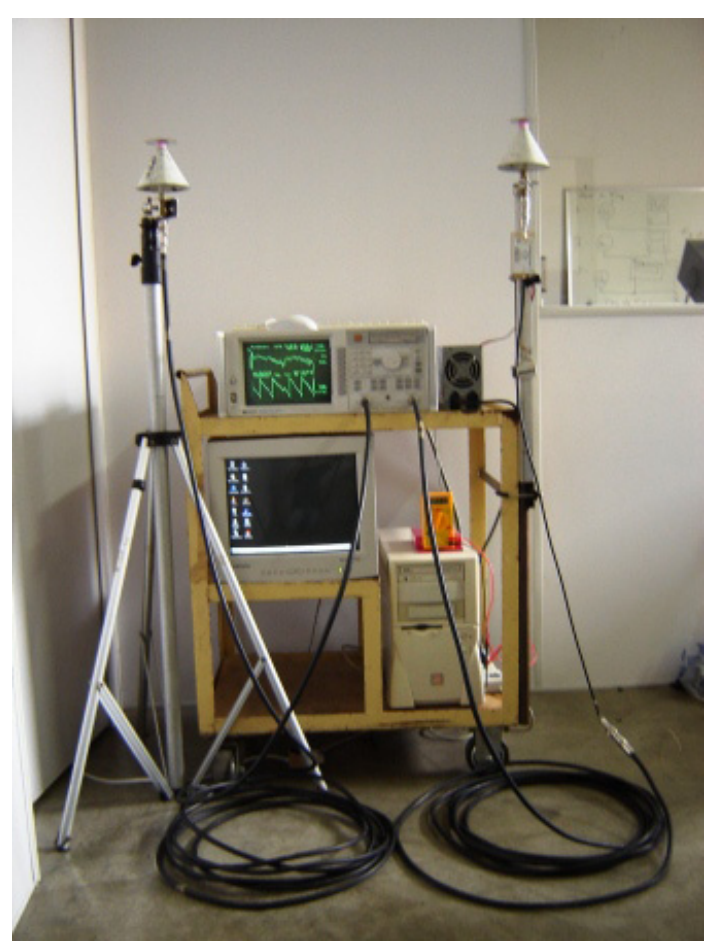

Figure 1 - Sounding System Source: [6]

Measurements of amplitude and phase of the channel transfer function along the time, $T(f, t)$, as used in [1], were carried out on 36 equally-spaced points set apart by $15 \mathrm{~cm}$ in a grid as shown in Fig.2 [6]-[7] which is the hall on the second floor of the Engineering building at Federal Fluminense University (UFF). The third and the ground floors were also sounded and the transmitter (TX) and the receiver (RX) were placed as much indoor as outdoor, in different positions [6]. In addition, three outdoor soundings were carried out near the Library and the Architecture College. Since the TX had to be connected to the $\mathrm{RX}$, only distances until $82 \mathrm{~m}$ could be processed in this sounding technique.

Table I - Channel sounding parameters

\begin{tabular}{|l|c|}
\hline \multicolumn{1}{|c|}{ Parameters } & Value \\
\hline Frequency Band $(\mathrm{MHz})$ & $960-1710$ \\
\hline Bandwidth $(\mathrm{MHz})$ & 750 \\
\hline Frequency resolution $(\mathrm{kHz})$ & 468.75 \\
\hline Delay resolution $(\mathrm{ns})$ & 1.33 \\
\hline Maximum delay $(\mathrm{ns})$ & 2133 \\
\hline Swept time (ms) & 696 \\
\hline Transmission Power $(\mathrm{mW})$ & 10 \\
\hline Antennas Gain $(\mathrm{dBi})$ & 2.14 \\
\hline Samples Number & 1601 \\
\hline
\end{tabular}




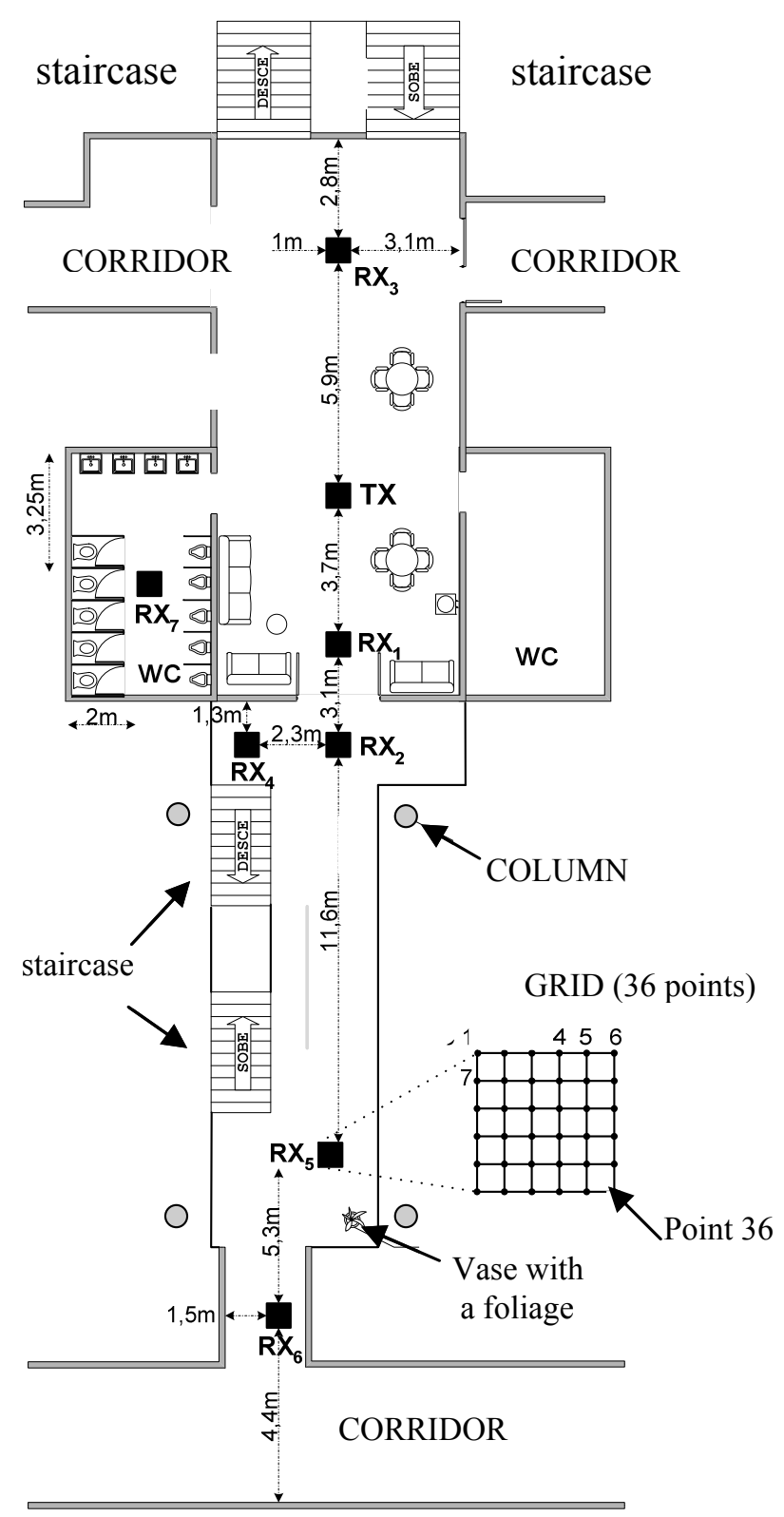

Figure 2 - Hall at second floor (Local 1) Source: [6]

Discone antennas were used for transmission and reception and the acquired data were stored in files of a computer through HPIB (Hewlett Packard Interface Bus) and a specific HP acquisition card.

\section{RESULTS AND COMMENTS}

Fig. 3 shows an example of a normalized PDP obtained by the inverse discrete Fourier transform applied on the windowed $T(f, t)$, after processing the data in an indoor-indoor scenario such as illustrated in Fig.1. The Blackman-Harris window with 3 (three) terms [8] was used and the noise threshold and the valid multipaths can be observed after de-noising a PDP with the CLEAN technique. The noise threshold was practically the same in all three techniques mentioned, about 60 $\mathrm{dB}$ below the peak of the PDP. However, it varied a little from one profile to another profile in the CFAR technique since the median varied. Valid multipaths did not overcome 350 ns. The mean delay spread was calculated for each environment and the results are shown in Table II and Table III.

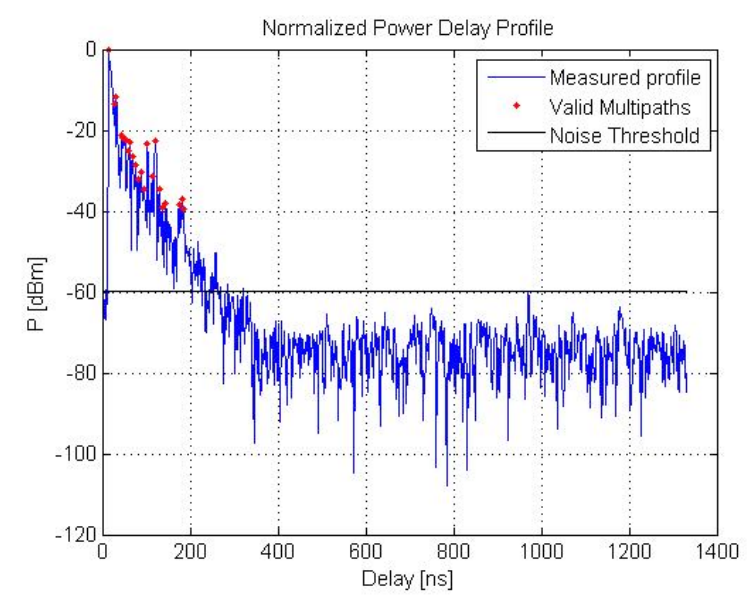

Figure 3 - Example of a PDP (local 1)

On Table II, Local 1 and 2 were on the second floor, Local 3 and 4 were on the third floor and Local 5 and 6 were on the ground floor of the Engineering building. The ground floors numbered 5 and 6 are wider than the others floors (1 - 4). At Local 1, 3 and 5, the TX was indoors while the RX was tested in several positions, and at Local 2, 4 and 6, the RX was indoors while the TX position was tested.

Table II - Mean delay spread in indoor-indoor, indoor-outdoor and outdoor-indoor channel

\begin{tabular}{|c|c|c|c|c|}
\hline Tech & Local & $\begin{array}{c}\text { Tx in } \\
\text { Rx in }\end{array}$ & $\begin{array}{c}\text { Tx in } \\
\text { Rx out }\end{array}$ & $\begin{array}{c}\text { Tx out } \\
\text { Rx out }\end{array}$ \\
\hline CFAR & & 12.75 & 14.45 & \\
\hline Clean & 1 & 13.40 & $\mathbf{2 5 . 6 6}$ & \\
\hline Wavel & & 13.61 & 15.06 & \\
\hline Dirty & PDP & 15.95 & 17.62 & \\
\hline CFAR & & 15.57 & & 18.51 \\
\hline Clean & 2 & 16.55 & & 18.18 \\
\hline Wavel & & 16.31 & & 19.01 \\
\hline Dirty & PDP & 18.88 & & 32.81 \\
\hline
\end{tabular}




\begin{tabular}{|c|c|c|c|c|}
\hline CFAR & & 15.85 & 14.52 & \\
\hline Clean & 3 & 19.47 & $\mathbf{2 1 . 1 6}$ & \\
\hline Wavel & & 16.43 & 15.38 & \\
\hline Dirty & PDP & 21.72 & 19.27 & \\
\hline CFAR & & 12.14 & & 14.83 \\
\hline Clean & 4 & 12.10 & & $\mathbf{2 0 . 3 1}$ \\
\hline Wavel & & 12.96 & & 16.30 \\
\hline Dirty & PDP & 15.12 & & 19.30 \\
\hline CFAR & & 23.63 & 26.61 & \\
\hline Clean & 5 & 22.78 & 27.15 & \\
\hline Wavel & & 23.89 & 22.05 & \\
\hline Dirty & PDP & 30.20 & 33.33 & \\
\hline CFAR & & 18.42 & & 22.69 \\
\hline Clean & 6 & 19.65 & & 26.60 \\
\hline Wavel & & 20.31 & & 24.82 \\
\hline Dirty & PDP & 23.99 & & 27.61 \\
\hline
\end{tabular}

In three floors of the building, with the TX indoor and RX outdoor, the mean delay spread was varying with the technique applied. Such variation was between 5 and 11.5 nanoseconds, with a maximum variation of $60 \%$. This situation shows the strongest influence of the de-noising technique in the delay spread. When the TX was outdoors and the RX indoors, the variation range of the delay spread was $0.5-5.5 \mathrm{~ns}$, with a maximum deviation of $30 \%$, so a smaller variation than the last situation, where the TX and RX were inverted.

On Table III, the TX and RX were outdoors: Local 7 was outside the library, in an open space with only one wall on one side; Local 8 was surrounded by trees and houses and Local 9 was also surrounded by trees and houses, but some trees were between the TX and the RX.

The delay spread calculated from the dirty profile, without any de-noising technique applied, has values close to that obtained with the CLEAN technique in general. In some environments, which delay spread is emphasized with bold numbers in Tables II and III, it was even smaller. It is probably due to the fact that the CLEAN technique discards all peaks that are under $80 \%$ of correlation, and perhaps this is not the better percentage of correlation to be used. Another fact can be that the reference signal used in this technique was not so pure [6].
Table III - Mean delay spread in outdoor channel

\begin{tabular}{|c|c|c|}
\hline Technique & Local & Tx out/ Rx out \\
\hline CFAR & & 18.97 \\
\hline CLEAN & 7 & $\mathbf{2 6 . 8 9}$ \\
\hline Wavelet & & 19.76 \\
\hline Dirty & PDP & 25.49 \\
\hline CFAR & & 23.76 \\
\hline CLEAN & 8 & 17.23 \\
\hline Wavelet & & 18.31 \\
\hline Dirty & PDP & 25.22 \\
\hline CFAR & & 14.84 \\
\hline CLEAN & 9 & $\mathbf{2 1 . 8 9}$ \\
\hline Wavelet & & 16.08 \\
\hline Dirty & PDP & 21.29 \\
\hline
\end{tabular}

In the situation where both TX and RX were outdoors, the delay spread was bigger than in the indoor situation, as we expected. Even though the environment was larger, it had trees and buildings around it [6], making all measurements behave as in a confined environment. Besides that, the sweep technique had limits because the TX and RX had to be connected. The maximum variation of the delay spread was about $7 \mathrm{~ns}$ in these environments, showing different results for each technique. However, when TX and RX were indoors, the delay spread was practically the same in any technique.

\section{CONCLUSIONS}

When no technique is used to de-noise the PDPs, delay spread is greater in general and it does not represent the real channel. In a project, this fact would result in lowering the transmission rate and also the system capacity, so the system would be bad dimensioned with overestimated values for the delay spread.

In this article delay spread was calculated from PDPs obtained from data available of wideband channel measured at UFF. For this, three denoising techniques were used and the mean delay spread was calculated for the denoised PDPs. It is worth to mention that the noise threshold considered is an important factor in the application of any technique, and it was kept practically constant in those used in this work.

The results show that the three different techniques here applied are equivalent when the 
sounded wideband channel is in an indoor environment, with TX and RX indoor, and they can be applied indistinctly. In all situations, however, the first (CFAR) and the third (Wavelet) techniques have led to similar values. In general the CLEAN technique showed bigger delay spreads and, sometimes, their values were worse than that obtained with the noisy profiles. Perhaps this technique must work with a different noise threshold or several thresholds depending on the PDPs.

So, the delay spread has some variation according to the de-noising technique applied to the PDPs, and presents similar results when the techniques are based on CFAR and wavelet. Future investigations must be realized in the noise threshold applied in the CLEAN technique. In parallel, another technique working with the RVM (Relevance Vector Machine) algorithm [9], that consists of a regression method based on Bayesian Statistics, is being studied in order to be applied in the identification of valid multipaths in a noisy PDP. Its main vantage is that no noise threshold is necessary to be considered.

\section{ACKNOWLEDGMENT}

To Federal Fluminense University - UFF/ Scientific Initiation Program (PIBIC).

\section{REFERENCES}

[1] D. Parsons, The Mobile Radio Propagation Channel, 2.ed, New York: John Wiley \& Sons, 2000.

[2] E.S. Sousa; V.M. Jovanovié, and C. Daigneault, "Delay Spread Measurements for the Digital Cellular Channel in Toronto", IEEE Trans. on Vehicular Technology, v. 43, pp. 837-847, November 1994.
[3] A.F. Molisch, "Ultra Wideband Propagation Channels - Theory, Measurements and Modeling”, IEEE Trans. on Vehic. Technology, v. 54, pp. 1528-1545, September 2005.

[4] M.H.C. Dias and G.L. Siqueira, "On the Use of Wavelet-Based Denoising to Improve Power Delay Profile Estimates from $1.8 \mathrm{GHz}$ Indoor Wideband Measurements", Wireless Personal Comm., Springer, v. 32, pp. 153-175, January 2005.

[5] B.H. Fleury, et al., "Channel Parameter Estimation in Mobile Radio Environments using the SAGE Algorithm", IEEE Mobile Radio Journal on Selected Areas in Communications v. 17, pp. 434450, March 1999.

[6] C.F. Souza, Análise da dispersão temporal de canais de banda ultralarga (UWB) através de medidas realizadas em ambientes internos e externos, Dissertação de Mestrado, 256 p., July 2006.

[7] C.F. Souza.; J.C.R. Dal Bello, and G.L. Siqueira, "UWB Systems in the Future 4G Mobile Telephony", Eleventh URSI Commission F Triennal Open Symposium on Radio Wave Propagation and Remote Sensing, October 2007.

[8] F.J. Harris, "On the use of Windows for Harmonic Analysis with the Discrete Fourier Transform", Proc. of the IEEE, v. 66, pp. 51-83, January 1978.

[9] D. Shutin; G. Kubin and B.H. Fleury,: "Application of the Evidence Procedure to the Estimation of Wireless Channels", EURASIP Journal on Applied Signal Processing, 23 p., 2007. 